\title{
Effect of Antihypertensive Drugs on Glucose Tolerance and Lipid Profile in Hypertensives With and Without Diabetes Mellitus
}

\author{
Authors \\ Dr M. Saboora Beegum ${ }^{1}$, Dr C.Edwin Emperor ${ }^{2}$, Dr Suja.S. $\mathbf{R}^{3}$ \\ ${ }^{1}$ Professor and Head, Department of Biochemistry, Government Medical College, Thiruvananthapuram \\ ${ }^{2}$ Associate Professor, Department of Plastic and Reconstructive Surgery, Kanyakumari Government Medical \\ College, Asaripallam, Nagercoil, Tamilnadu \\ ${ }^{3}$ Assistant Professor, Dept of Biochemistry, Indira Gandhi Government Medical College, \\ Thiruvananthapuram
}

\section{Introduction}

Diabetes is the most common endocrine disease. It is a heterogeneous primary disorder of carbohydrate metabolism with multiple etiologic factors that generally involve absolute or relative insulin deficiency or insulin resistance or both. All causes of Diabetes Mellitus lead to hyperglycemia, which is the hail mark of this disease syndrome.

In all types of Diabetes Mellitus, the glucose tolerance is reduced. The theory of glucose tolerance is that a normal person should be able to remove a glucose load from plasma with in a short specified period of time. If for some reason he is unable to do so, the plasma concentration remains elevated longer than normal and the patient is said to have reduced glucose tolerance.

Hypertension is defined as elevated blood pressure. It is impossible to define with precision the limits of "normal" B.P. The B.P of a given individual varies widely over time, depending on many variables, including sympathetic nervous system activity, posture, state of hydration and skeletal muscle tone. Accordingly any definition for hypertension is arbitrary.

Hypertension and Diabetes Mellitus are chronic medical conditions that frequently co-exist. In the United States, it is estimated that 10 million persons are suffering from Diabetes Mellitus, 60 million from hypertension, and 3 million from the combination of the two. There may be a causal relationship between hypertension and Diabetes Mellitus. Obesity may be a precipitating factor for Hypertension and Non-Insulin-DependentDiabetes Mellitus. Those with Insulin-DependentDiabetes-Mellitus generally became hypertensive with the onset of nephropathy. Glucose intolerance, insulin resistance, and hyperinsulinemia may be aggravated by hypertensive therapy, especially with diuretic and beta blockers. It has been estimated that hypertension is approximately twice as common in the Diabetics as in the non-diabetic patients.

Antihypertensive therapy presently available include Diuretics, Beta blockers, Alpha blockers, Calcium channel blockers, Angiotensin- 
converting enzyme inhibitors. Almost all diuretics and beta blockers induce abnormal glucose tolerance. There is no evidence that alpha blockers, ACE inhibitors or calcium channel blockers cause glucose intolerance. Diuretics and beta blockers have relative contra indication, where as alpha blockers, calcium channel blockers and ACE inhibitors are recommended as first line of monotherapy.

Several drugs used for antihypertensive therapy interact with lipoprotein metabolism and increase associated coronary risk factors. Beta blocker monotherapy with cardio-selective and non cardio-selective beta blockers without intrinsic sympathomymetic activity usually increase serum triglycerides and decrease the serum concentration of high density lipoprotein especially $\mathrm{HDL}_{2}$ cholesterol. Beta blocker monotherapy has little influence on serum cholesterol or low density lipoprotein cholesterol concentration.

In addition the antihypertensive drug beta blocker produce adverse change in blood lipds. Of the alternative first line antihypertensive agents, the calcium channel blockers and ACE inhibitor drugs are lipid 'neutral' but only alpha-1-blockers have favourable effect on the lipid profile.

\section{Effect of Various Drug Classes on Serum Lipids and on Glucose Tolerance}

\begin{tabular}{|l|l|c|c|c|c|}
\hline $\begin{array}{l}\text { Sl. } \\
\text { No. }\end{array}$ & Drug class & Triglyceride & Cholesterol & HDL & $\begin{array}{c}\text { Glucose } \\
\text { Tolerance }\end{array}$ \\
\hline 1. & Thiazide diuretic & $\uparrow \uparrow \uparrow$ & $\uparrow$ & 0 & Decreased \\
\hline 2. & Beta Blockers (Non selective) & $\uparrow \uparrow \uparrow$ & 0 & $\downarrow$ & Decreased \\
\hline 3. & Calcium channel blocker & 0 & 0 & $\uparrow$ & Unchanged \\
\hline 4. & ACE inhibitor & 0 & 0 & & Increased \\
\hline 5. & Alpha Antagonist & 0 & 0 & & Increased \\
\hline
\end{tabular}

\section{Review of Literature}

\section{Effect of beta-blockers on Lipid Profile}

Several drugs used for antihypertensive therapy may interact with lipoprotein metabolism and increase associated coronary risk factors. Betablocker monotherapy with cardio selective or noncardioselective beta-blocker without Intrinsic Sympathomimetic Activity (ISA) usually increase serum triglyceride and decreases the concentration of HDL cholesterol especially HDL2 Cholesterol. With the exception of non- cardioselective beta-blocker sotalol, beta-blocker therapy has little influence on serum total cholesterol or LDL cholesterol. Two beta-blockers possessing ISA, acebutolol and pindolol did not increase serum triglycerides, total cholesterol or LDL cholesterol. Acebutolol produced a non-significant decrease in HDL cholesterol while pindolol elevated HDLcholesterol and the ratio of HDL-cholesterol to total cholesterol. Beta-blockers with the exception of pindolol, decreased the free fatty acid concentration.

Beta-blocker therapy has no effect on lipoprotein lipase activity but enhances LCAT activity^].

\section{Eeta-Blockers}

Beta-blockers with significant adverse effect on triglycerides and HDL-cholesterol include propranolol, metoprolol and atenolol. Betablockers with intrinsic sympathomimetic activity (ISA) such as pindolol in hypertensive subjects and celiprolol in hypertensives with NIDDM have been shown to possess no adverse effect on lipid or carbohydrate metabolism. Part of the adverse effect of beta-blockers on lipid metabolism is due to their effect on lipoprotein lipase activity and LCAT activity. Beta-blockers possess a positive effect on coronary disease mortality in secondary prevention trials Us]. In a risk factor study, patients on antihypertensive therapy lower HDL and higher triglycerides. In hypertensive patients treated for 10 years with combination of diuretics, beta-blockers and hydralazine often developed overt' diabetes than normotensive subjects. Use of diuretics and beta-blockers in .hypertensives with NIDDM has been associated with elevated glucose, triglycerides and VLDL and lowered HDL. 
The design and early development of drugs which inhibited Angiotensin Converting Enzyme Inhibitor (ACEI) was conducted with the expectation that the principal applications of such agents would lie in clinical syndromes in which the activity of the renin angiotensin system was enhanced - for example renovascular hypertension and certain varieties of cardiac failure. With the introduction of the first orally active ACE inhibitor, captopril, it became apparent, however that such treatment was applicable to essential hypertension, in which both the hypertensive effect and the acceptability of ACE inhibitors were found to be at least comparable with those of other drugs. The use of ACE inhibitors either given alone or in combination with other drugs, in uncomplicated essential hypertension now constitutes a major indication and has appreciably increased therapeutic freedom.

\section{Monotherapy using ACE inhibitor}

Even in uncomplicated essential hypertension, circulating plasma concentrations of angiotensin II are within a range in which they exert an immediate, direct effect on the arterial pressure. Thus acute blockage of the renin-angiotensin system, as by infusing an angiotensin II antagonist such as saralasin or by inhibiting ACE, cause an immediate reduction of both arterial pressure and plasma aldosterone in proportion to the previously ..prevailing plasma angiotensin II concentration. In essential hypertension, however, circulating concentration of renin and angiotensin II are usually modest or low and decline further with age. The ACE inhibitors are reported to be as effective as diuretics, beta-blockers, calcium antagonists and methyl dopa. In a double blind trial, Herrick et al showed enalapril has much more antihypertensive effect than atenolol.

\section{Diuretics, Beta-blockers and ACE inhibitors}

Examining a range of drugs added to the treatment of patients whose blood pressure was inadequately controlled by thiazide and beta-blocker, Bevan et al reported captopril to be more effective than nifedipine or hydralazine. From various studies captopril was the most effective third drug in comparison with methyldopa, prazosin, hydralazine and nifedipine.

\section{Object and Scope of Study}

1. Long term treatment of hypertensives with antihypertensive drugs can induce diabetes mellitus. Hyperglycemia and insulin resistance of treated hypertensives could cause diabetes mellitus.

2. In the present study, the beta blockers and Angiotensin Converting Enzyme Inhibitors (ACEI) have been compared to assess their effects on glucose tolerance and lipid profile in hypertensives and in hypertensive diabetic patients. With the above objectives the following project is proposed.

3. The drug administration will be for two periods of treatment [ (i) 6 months to 1 year and (ii) 2 years] ACE inhibitor and Beta-blocker will be given to hypertensives with and without diabetes for the above periods. Two groups of patients with age 31-40 years and 41-50 years will be included in the study.

Biochemical investigations include

a) Oral glucose tolerance after a glucose load of $1 \mathrm{gm} / \mathrm{kg}$ body weight

b) Lipid profile

1. Total cholesterol

2. Triglycerides

3. LDL cholesterol

4. HDL cholesterol

\section{Materials and Methods}

The patients included in this study were those attending the Diabetic and Hypertensive clinics of Medical college Trivandrum. 200 cases were studied, all members of this study group were non obese, non smoking and non alcoholic with' no family. history of diabetes. All of them were between 31 - 50 years of age. 
The control group included 10 healthy non obese, non smoking, non alcoholic volunteers with no family history of Diabetes Mellitus for each age group 31-40 and 41-50.

Mainly 3 groups of patients were studied.

1. Patients with Diabetes Mellitus alone

2. Patients with Diabetes Mellitus and Hypertension

3. Patients with Hypertension alone

The Diabetic patients included in this study were those on oral hypoglycemic drug (Sulphonylurea). Hypertensive patients in this study were on monotherapy either with Propranolol or with Angiotensin-Converting Enzyme Inhibitors.

For assessing glucose tolerance the patients were divided in to subgroups according to age and duration of anti hypertensive treatment.

Depending on age 1) Between 31 - 40 Years.

2) Between 41 - 50 Years.

Depending on duration of treatment

1) 6 Months to 1 Year treatment,

2) 2 Years treatment.

Two types of antihypertensive drugs were studied.

1.Betablocker (Propranalol / 30-60mg/day)

2.Angiotensin Converting Enzyme inhibitor.

(ACE inhibitor) Enalapril 5 - $10 \mathrm{mg} /$ day

Thus 10 subgroups of patients with a sample number 10 were studied in each age group (31-40 years and 41-50 years).

1. Normal between ages 31 - 40 years and 41-50 years

2. Patients with Diabetes Mellitus alone

3. Patients with Diabetes Mellitus and hypertension on beta blocker 6 months 1 year treatment

4. Patients with Diabetes Mellitus and Hypertension on betablocker 2 Years treatment

5. Patients with Diabetes Mellitus and Hypertension on ACE Inhibitor for 6 months to 1 year treatment

6. Patients with Diabetes Mellitus and Hypertension on ACE inhibitor for 2 years treatment
7. Patients with Hypertension on betablocker 6 months to 1 year treatment

8. Patients with Hypertension on betablocker 2 years treatment

9. Patients with Hypertension on ACE Inhibitor for 6 months to one year treatment

10. Patients with Hypertension on ACE Inhibitor for 2 years treatment

Lipid profile was assessed in six groups of patients irrespective of their age and duration of treatment.

1. Normal 31 - 50 Years

2. Patients with Diabetes alone

3. Patients with Diabetes and Hypertension on Beta blocker

4. Patients with Diabetes and Hypertension on ACE inhibitor

5. Hypertensive patients on Beta-blocker

6. Hypertensive patients on ACE inhibitors. 


\section{Results and Discussion}

Table I Details Of Glucose Tolerance Test Done In Normals And In Diabetics (Age Group : 31 - 40 Yrs) Values are given in milligram percentage + SD.

\begin{tabular}{|l|l|l|l|}
\hline Time & Nomal $(31-40$ yrs) & Diabetes (31-40 Yrs) & P V a lue \\
\hline Fasting & $76 \pm 6$ & $128 \pm 11$ & $<<0.005$ \\
$0.5 \mathrm{hr}$ & $137 \pm 7$ & $210 \pm \pm 11$ & $<<0.005$ \\
$1.0 \mathrm{hr}$ & $160 \pm 8$ & $275 \pm 13$ & $<<0.005$ \\
$1.5 \mathrm{hr}$ & $122 \pm 5$ & $248 \pm 66$ & $<<0.005$ \\
$2.0 \mathrm{hr}$ & $87 \pm 4$ & $210 \pm 10$ & $<<0.005$ \\
$2.5 \mathrm{hr}$ & $72 \pm 8$ & $178 \pm \pm 10$ & $<\mathrm{v} 0.005$ \\
\hline
\end{tabular}

Glucose tolerance is significantly lowered in all Diabetes.

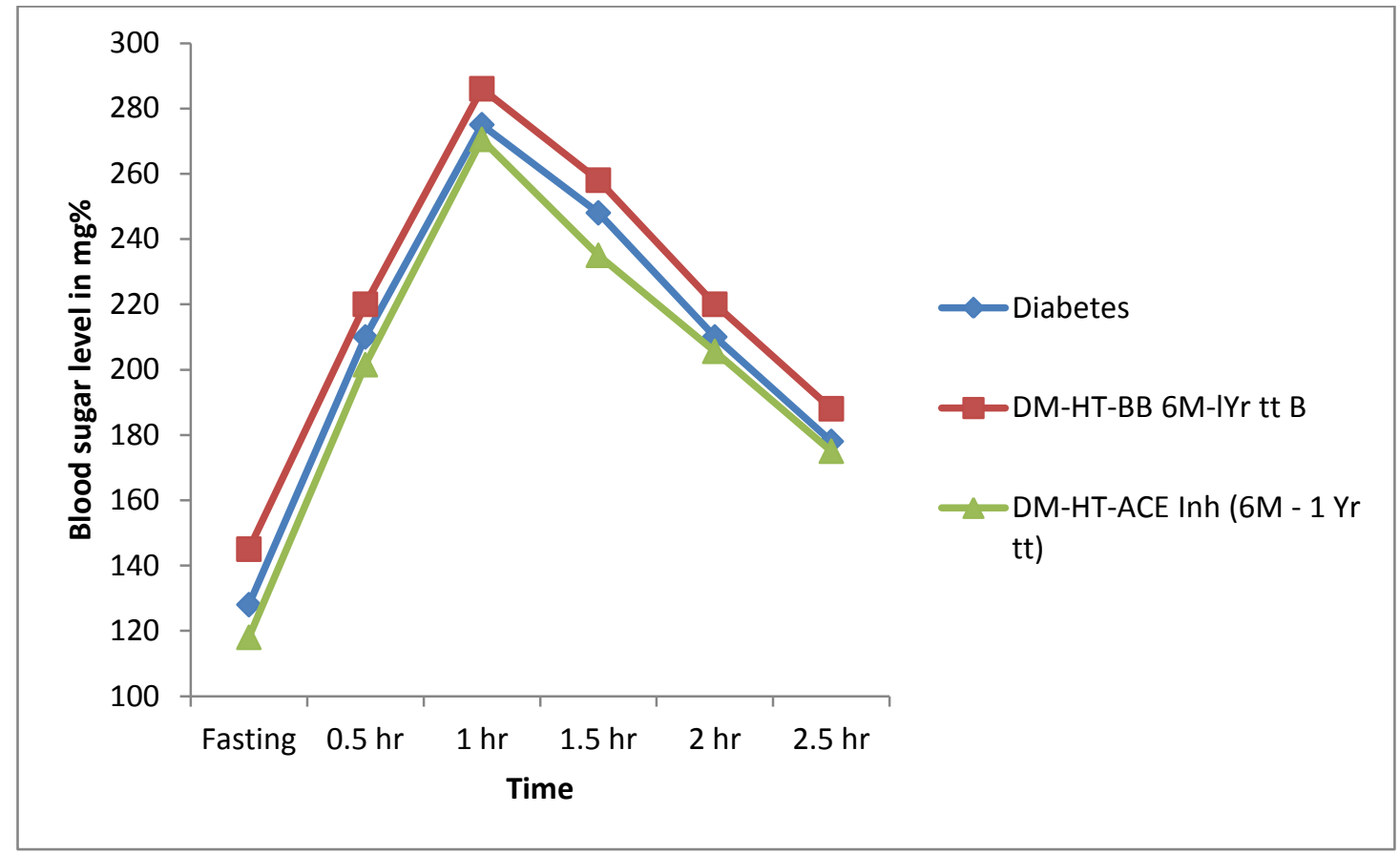

In the present study the comparison of the effects of antihypertensive agents for different periods, 6 months to 1 ear and 2 years in hypertensive patients. Beta blocker therapy Lowered the glucose tolerance in the initial phase of therapy itself. No further deterioration could be seen on continuing the therapy for a longer period of 2 years. The difference in blood glucose levels between the two different periods of therapy, namely $\mathrm{E}$ months to 1 year and 2 years, is statistically not significant (Table VIII) The reduction in glucose tolerance by the beta blockers is observed in initial period of therapy only. Whether any metabolic changes that occured secondary to the initial intolerance to glucose by the beta-blockers could nullify or neutralise and prevent a further drop in glycemic control on ntinued therapy with beta-blockers cannot be said from the present study. Such a possibility exists since long term therapy does not elevate the blood glucose level. 


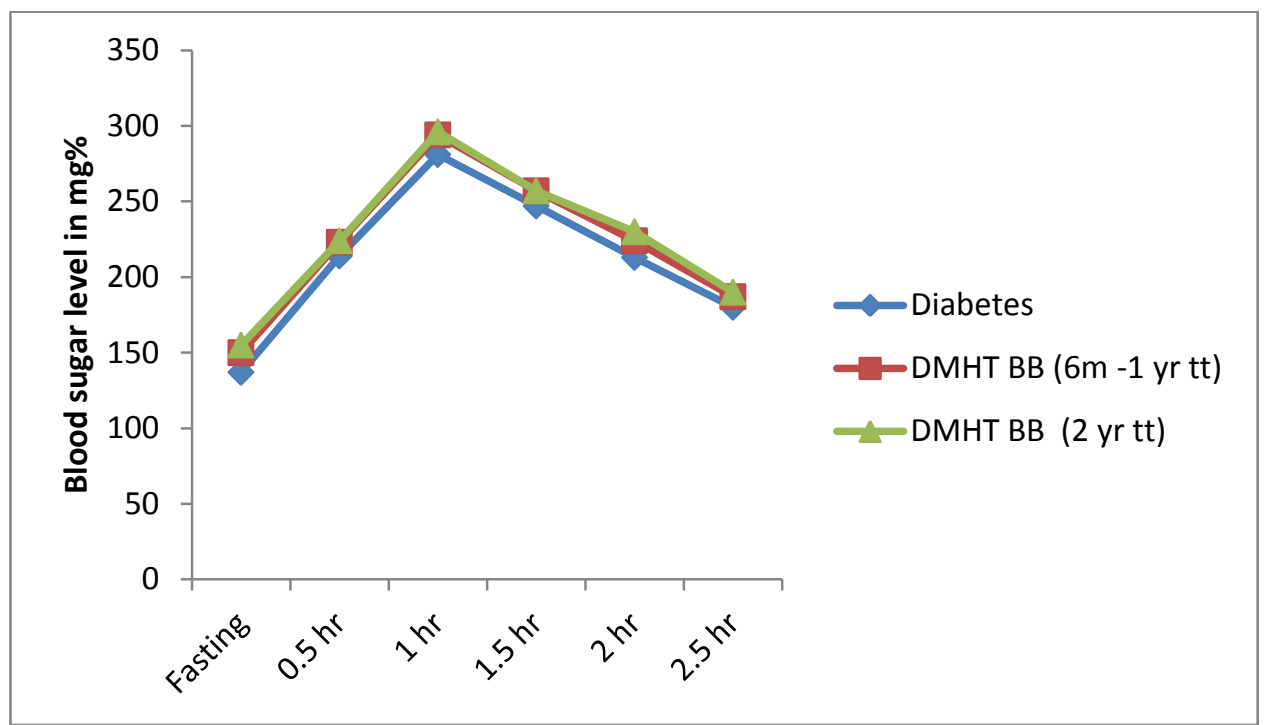

Fig. (14). Glucose tolerance test done in diabetics and in diabetic hypertensive on BB for $6 \mathrm{~m}-1 \mathrm{yr}$ treatment.

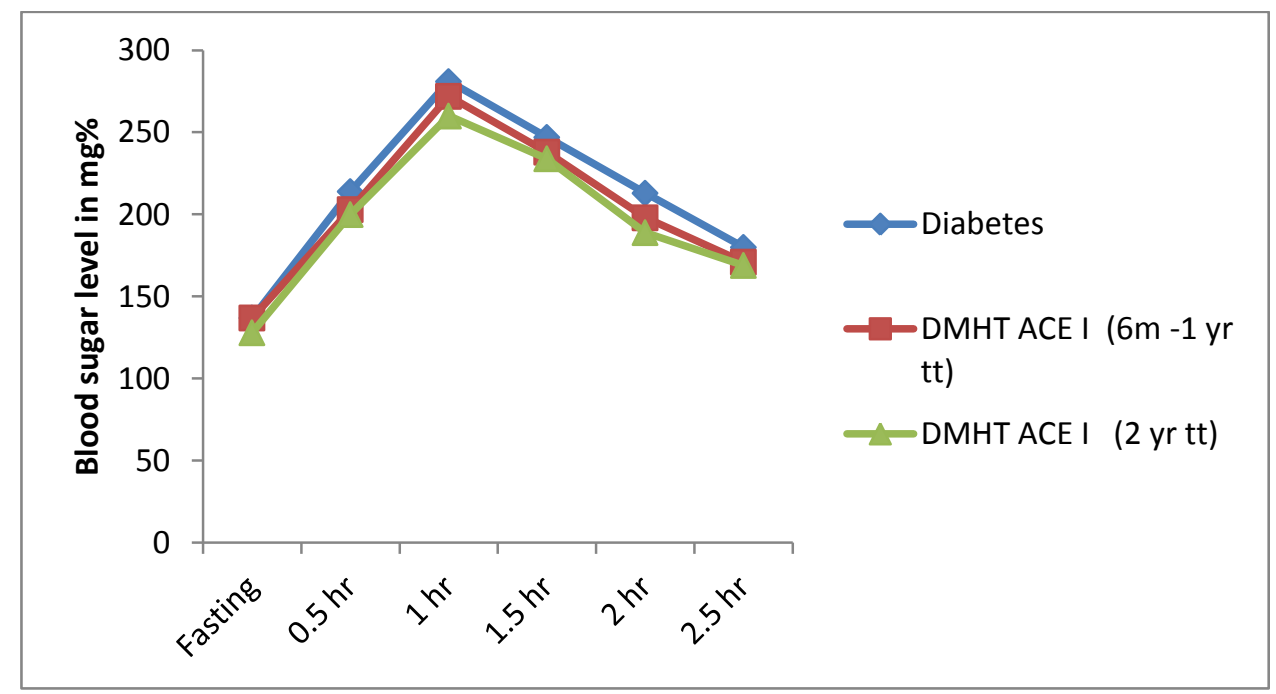

Fig. (15). Glucose tolerance test done in diabetics and in diabetic hypertensives on ACEI for $6 \mathrm{~m}-1 \mathrm{yr}$ and for 2 yrs treatment. (age group 41-50 yrs).

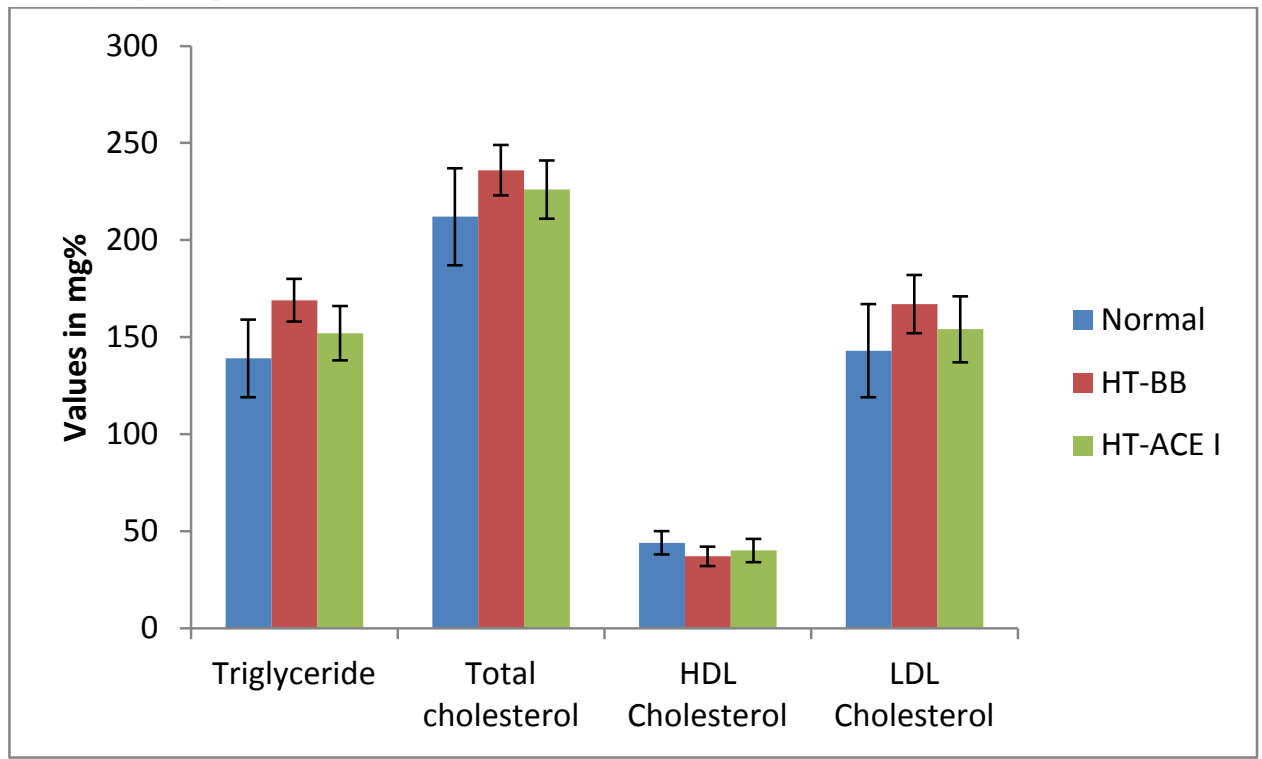

Fig. (20) Lipid profile in normals, HT on ACEI and HT on BB 


\section{Triglyceride}

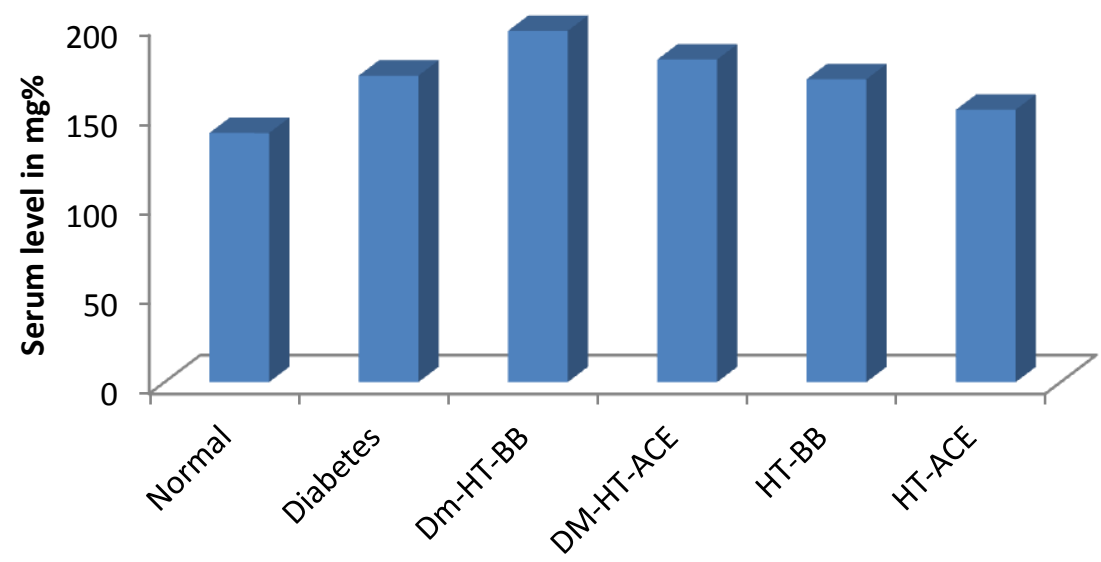

Fig (21)

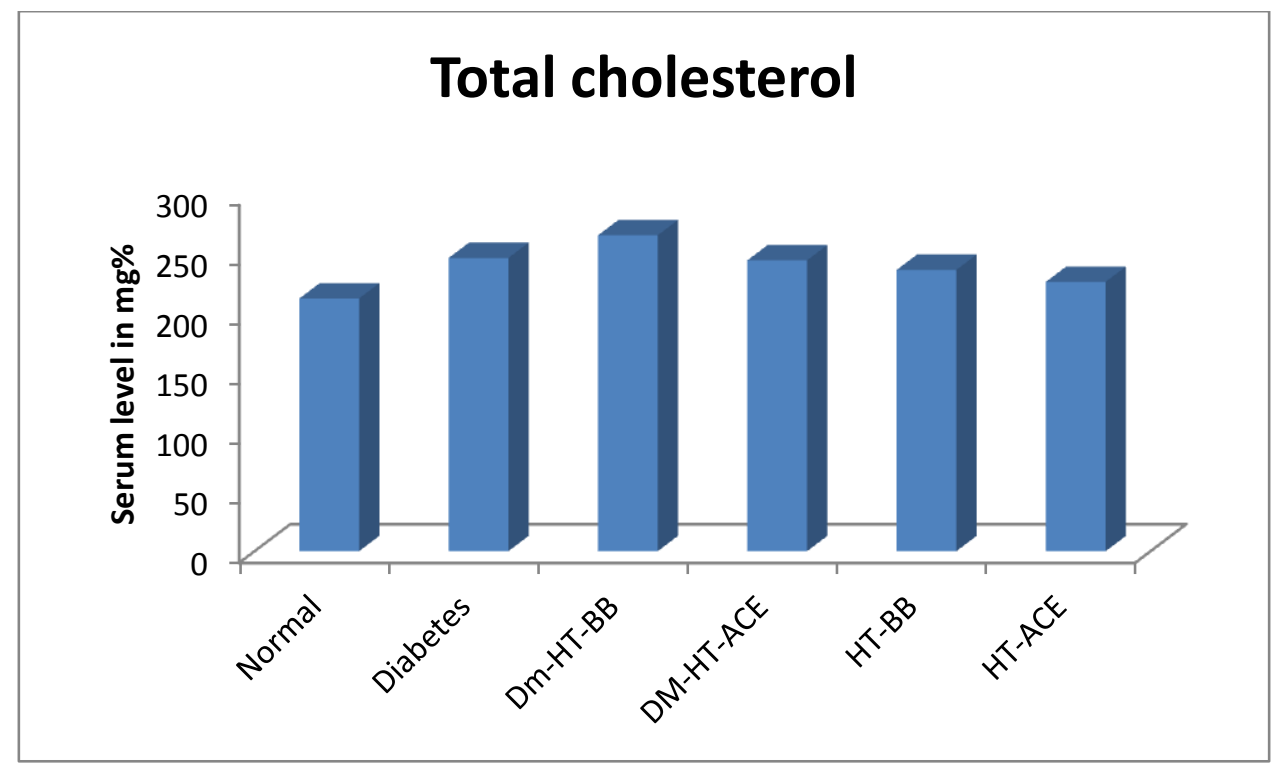

Fig (23)

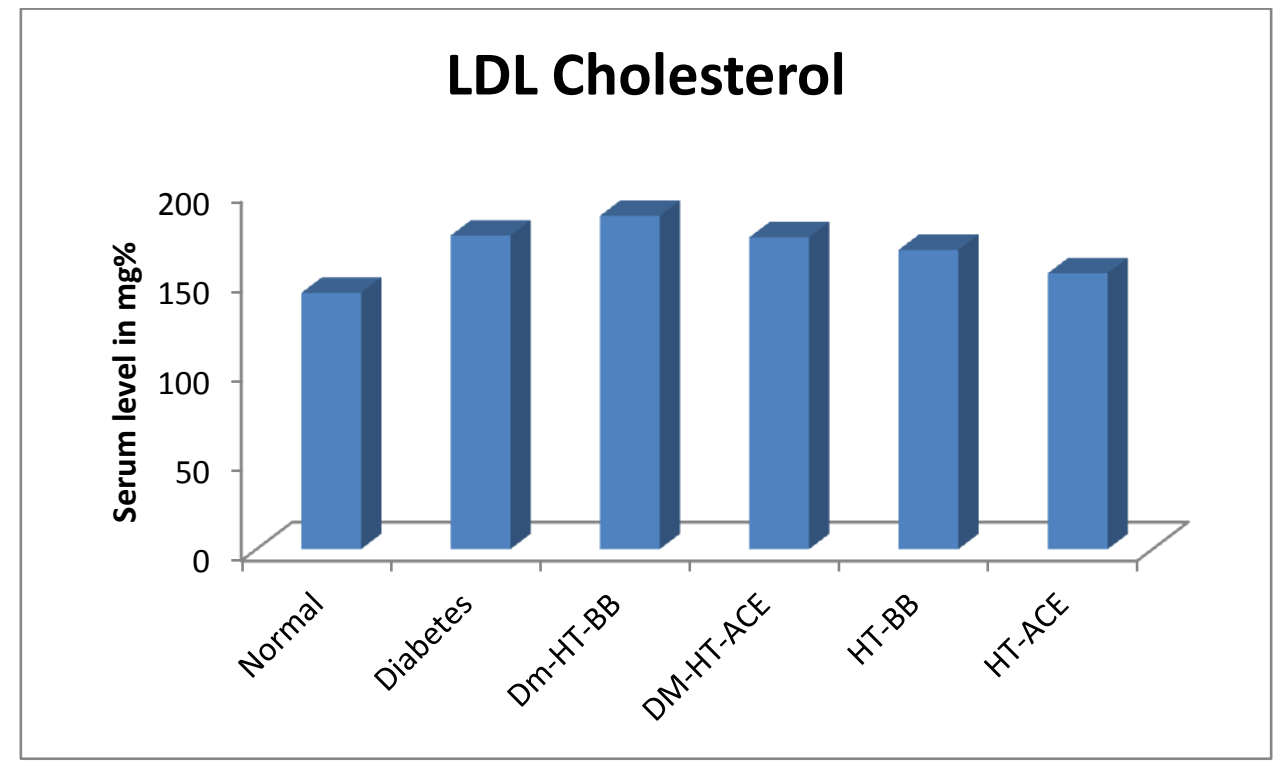




\section{Summary and Conclusion}

The present study carried out involves six separate groups. First group consisted of normal individuals without any symptoms or complaints of diabetes mellitus or of hypertension. The second group included only diabetes mellitus patients. Diabetic patients with hypertension are divided into two groups. One group received beta blockers, and the other ACE inhibitors which formed third and fourth groups respectively. The fifth and sixth groups form the hypertensive patients receiving beta blockers and ACE inhibitors respectively.

The first phase of the study consisted of the evaluation of biochemical parameters at the end of 6 months to one year of drug administration. In the second phase, the biochemical assessment is done at the end of 2 years treatment with the hypotensive agents. All the patients and normals belong to the age group 31 to 40 years.

In a second trial, the whole of the above schedule is repeated in normals and in patients of the age group 41-50 years. The results of the study is as given below.

1. All diabetic patients included in the study showed low glucose tolerance as compared to normals.

2. Treatment with beta blocker for 6 months to 1 year lowered the glucose tolerance while ACE inhibitor given for the same period improved the glucose tolerance in hypertensive diabetic patients. Similar pattern is seen on continuing the treatment regime for two years.

3. Treatment with beta blocker for six months to one year lowered the glucose tolerance in diabetic hypertensive subjects. No further deterioration is observed on continuing the treatment for two years. The glycemic control of diabetic hypertensives is improved on ACE inhibitor therapy for 6 months to one year and further improvement on continuing the treatment for 2 years is only marginal.
4. Beta blocker besides being antihypertensive appeared to be hyperglycemic too while ACE inhibitor appears to possess both hypotensive and hypoglycemic effects. The same pattern is observed when tested for 2 periods- 6 months to one year and for 2 years.

5. Hyperglycemic effect of beta blocker observed in the 1st phase of treatment did not aggrevate in the second phase. But the hypoglycemic effect of ACE inhibitor is slightly more in the second phase.

6. Glucose tolerance is age dependent in both normals and diabetic patients.

7. Hyperglycemic effect of beta blocker and hypoglycemic effect of, ACE inhibitor are more prominent in the second phase (2 years of treatment) in hypertensive diabetics of age goup 41-50 years. However, the values at the end of two years are not statistically significant when compared to that of short term period.

8. The fact that hypotensive property of beta blocker is associated with hyperglycemic effect is further substantiated by performing oral glucose tolerance test in hypertensive patient obtaining the beta blocker for 6 months to one year. ACE inhibitor administration to hypertensives for the same period does not alter the glucose tolerance. However, a mild hypoglycemic effect of ACE inhibitor is evident from the results of the oral glucose tolerance test conducted after the 2 years of treatment.

9. The hyperglycemic effect of beta blocker observed in the inital phase of 6 months to one year is not aggravated by continuing the treatment for 2 years in hypertensives.

10. Lipid profile is altered in diabetes as compared to normals. The altered lipid profile is changed adversely indicating the increased risk for CAD by treatment with beta blocker in hypertensive diabetic patients. 
11. ACE inhibitor does not affect the lipid profile in diabetic hypertensive subjects.

12. Both the beta blocker and ACE inhibitor alter the lipid profile of hypertensives as compared to normotensive controls but two different degrees. The increase in blood lipid constituents in beta blocker treated patients is statistically significant while that in ACE inhibitor treated patients it is statistically insignificant.

13. Serum triglycerides are elevated by beta blocker and ACE inhibitor treatment in hypertensives with and without diabetes. But the increase is more marked in case of beta blocker treatment compared with ACE inhibitor treatment.

14. Serum total cholesterol and LDL cholesterol of hypertensive diabetics is unchanged by ACE inhibitor but is elevated by beta blocker. But in hypertensives total cholesterol and LDL cholesterol are markedly elevated by beta blocker but only marginally increased by ACE inhibitor.

15. Serum HDL cholesterol of hypertensive subjects is reduced to a greater extent by beta blocker as compared to that by ACE inhibitor. It is unaffected in diabetic hypertensives by ACE inhibitor but is lowered by beta blocker.

16. In diabetic hypertensives the ratio of HDL cholesterol to LDL cholesterol is unaffected/slightly improved by the ACE inhibitors; but it is lowered with beta blocker therapy.

17. Both beta blocker and ACE inhibitor lowered the HDL - 111 cholesterol ratio in hypertensives, but only marginally ir. ACE inhibitor treated patients and markedly in beta blocker treated patients compared to the normals.

It is concluded that ACE inhibitor is the drug of choice in hypertensives and hypertensives with diabetes mellitus. $Z z$ is a better hypotensive agent compared to beta blocker especially ir. hypertensives with diabetes mellitus since it possesses hypoglycemic effect and no significant effect on lipid profile. In hypertensives it is superior to beta blocker since it does not affect the glucose homeostasis and produces only marginal increase in total cholesterol, triglycerides, LDL cholesterol and minimal reduction in HDL cholesterol. These could be taken to predict that there is only minimal risk towards atherogenesity in diabetics with hypertension and in hypertensives.

\section{References}

1. Hamilton BP Am. J. of Kidney Disease. 1990 Oct; 16(4 Suppl 1); p 20-9 1986 Mark C Houston

2. Treatment of hypertension in Diabetes Mellitus American Heart J. 118 : 819829, 1989.

3. Effect of Beta Blocker on lipid profile Am. Heart J. 109 : 1192-6, 1985.

4. Jc Fruchart, J Shepherd Clinical Biochemistry, Principles Methods and application. Human Plasma Lipoprotfien; Dys-lipoproteinemoia in D M, P174

5. Antihypertensive drugs and blood lipids J. Human Hypertension, 4 Suppl. 3 ; 17, 1990 Octo

6. Peter E Pool. Progress in Cardio Vascular disease Vol 35 No.1, 1993

7. Michael W Steffes Clinical Chemistry 32 : 10(B), 1986.

8. Classification of Diabetes Mellitus, W.H.O., 1985

9. Detection and Management of lipid disorders in Diabetes consensus statement. Diabetes care Vol. 18, Supp I Jan 1995.

10. Drury. PL Diabetes and arterial hypertension Diabetologia. 24 : 1-9, 1983

11. P, Lombrail. P, Eschwego. E, Canirel. J Prevalence do hypertension arterielle chez le diabetigue, Influence des traitements antihypertenseurs sur le 
controle metaboligue Arch Mai Coeur 76 : 169-71, 1983

12. Havlik. RJ, Hubert. HB, Fabsitz. RR, Feinleib. M Weight and hypertension Ann Intern Med. 98 : 855-859, 1983

13. Parving HH, Anderson AR, Smidt UM, Christiansen JS, Oxenboll, Svendsen PA Diabetic nephropathy and arterial hypertension : the effect of antihypertive tt in diabetes 32 (Supp 2) 83-87, 1974

14. Garcia. $M J$, $M c$ Namara. PM, Gordon. T, Kannel. WB Morbidity and mortality in diabetes in the Framingham population Diabetes $23: 105-114,1974$. 